\title{
Advances in Exercise, Physical Activity, and Diabetes Mellitus
}

\author{
Sepideh Mohajeri and Michael C. Riddell
}

\section{Introduction}

M ORE AND MORE EVIDENCE SUGGESTS THAT REGULAR EXERCISE is the single best lifestyle tool to prevent, delay, or treat diabetes. Emerging research is allowing clinicians and patients to choose the best forms of exercise and helping us all understand the mechanisms for why exercise is so beneficial. Motivating patients to be more physically active and outlining strategies to help prevent exercise-associated hypoglycemia in type 1 diabetes remain the two most challenging aspects of the physical activity prescription. This year, we highlight eight leading peer-reviewed original articles on exercise, physical activity, and diabetes that were published in the period July 1, 2013, to June 30, 2014. From the emergence of exercise smart pumps to the importance of postmeal walking, this selective review covers some of the key advances made over the past year.

\section{Blood glucose responses to type, intensity, duration, and timing of exercise}

Colberg $S R^{1}$, Hernandez $M J^{2}$, Shahzad $F^{2}$

${ }^{I}$ Old Dominion University, Norfolk, VA; and ${ }^{2}$ Diabetes Hands Foundation, Berkeley, CA

Diabetes Care 2013; 36: e177

\section{Background and Rationale}

To encourage involvement in regular exercise in the management of diabetes, the Diabetes Hands Foundation initiated the online Big Blue Test (BBT) data collection program. During the BBT, individuals with diabetes partake in physical activity and record blood glucose (BG) levels with the hopes of raising awareness regarding the benefits of exercise. This study uses data from the 2012 BBT to assess how various parameters of exercise (type, intensity, duration, and time since last meal) impact glycemia.

\section{Methods}

A total of 5,157 individuals with diabetes, of which approximately $90 \%$ administered insulin, used an iPhone app or the BBT website to report their category of exercise, and BG values prior to and immediately following $\geq 1$ physical activity session(s). Different forms of exercise were determined by looking at a past BBT event. Reported categories included exercise type (walking, running/jogging, cycling, conditioning machines, dancing, nonspecified), intensity (moderate or vigorous), duration $(\leq 10,11-19,20-29$, or $\geq 30 \mathrm{~min})$, and the amount of time passed since previous meal ingestion (30 min, 1,2 , or $\geq 3 \mathrm{~h}$ ).

\section{Results}

In order from most to least popular, participants reported engaging in the following activities: walking $(48.5 \%)$, nonspecified (18.7\%), running/jogging (11.9\%), cycling (8.8\%), conditioning machines $(6.4 \%)$, and dancing $(5.7 \%)$. These activities and other types of exercise promoted decreases (75.8\% of cases) and increases (15.4\%), or resulted in unchanged $(8.8 \%)$ BG levels. The smallest decrease in BG was seen with walking $(-25.0 \pm 42.4 \mathrm{mg} / \mathrm{dL})$, while the largest change was observed with cycling activities $(-42.4 \pm$ $48.8 \mathrm{mg} / \mathrm{dL}$ ). Interestingly, moderate forms of exercise decreased BG levels $(-32.7 \pm 44.1 \mathrm{mg} / \mathrm{dL})$ more than vigorous activity $(-28.0 \pm 53.6 \mathrm{mg} / \mathrm{dL})$. Exercise of longer duration led to a greater reduction in $\mathrm{BG}$ when all exercise intensities were combined, and with moderate intensity alone, but not with vigorous exercise intensity alone. The greatest decrease in BG occurred when exercise was completed 1 or $2 \mathrm{~h}$ after a meal (similar decline in both periods), when compared with $30 \mathrm{~min}$ or $\geq 3 \mathrm{~h}$ postmeal.

\section{Discussion}

Engaging in various types, durations, and intensities of exercise, at different postprandial time points, can all impact the glucose response. While exercise generally decreases BG, high-intensity exercise-associated counterregulatory hormones can help to prevent hypoglycemia or may even cause glucose to rise (1). As such, individuals with type 1 diabetes may find it beneficial to incorporate a $10 \mathrm{~s}$ sprint before or after moderate activity to help limit the drop in glycemia. Longer bouts of exercise increase the likelihood of

School of Kinesiology and Health Science, York University, Toronto, ON, Canada. 
low BG (2), making it necessary to be well-prepared to treat hypoglycemia. Finally, when planning an exercise session, it can be beneficial to consider the length of time that has passed since a meal to optimize the desired decrease in BG, or to better avoid the risk of postexercise hypoglycemia.

\section{Comment}

As a part of a healthy lifestyle, individuals with diabetes can engage in a variety physical activity modalities. However, predicting the $\mathrm{BG}$ response to exercise is not always easy. This article shows that there is no single response to exercise; many variables can be manipulated to achieve the desired glycemic response from an exercise session. A basic understanding of how different types of moderate and vigorous forms of exercise can impact BG levels based on this large database should help individuals anticipate their body's reaction and therefore better plan treatments or schedule exercise sessions. While the results of this article are based on self-reported data, providing in-depth information regarding responses to exercise type, duration, intensity, and time can help clinicians to provide accurate exercise prescriptions. Also based on this data set, it appears that insulin users have much more of a drop in glycemia with most forms of exercise compared with nonusers (3). Physicians can use this information to better utilize physical activity as a viable therapeutic option for high blood glucose in patients with diabetes.

\section{Reduced hypoglycemia and increased time in target using closed-loop insulin delivery during nights with or without antecedent afternoon exercise in type 1 diabetes}

Sherr $J^{1}$, Cengiz $E^{1}$, Palerm $C C^{2}$, Clark $B^{2}$, Kurtz $N^{2}$, Roy $A^{2}$, Carria $L^{1}$, Cantwell $M^{2}$, Tamborlane $W V^{1}$, Weinzimer $S A^{1}$

${ }^{I}$ Department of Pediatrics, Yale University School of Medicine, New Haven, CT; and ${ }^{2}$ Medtronic Diabetes, Northridge, $C A$

Diabetes Care 2013; 36: 2909-14

This manuscript is also discussed in article on Diabetes Technology and Therapy in the Pediatric Age Group, p. S-103.

\section{Background and Rationale}

Individuals with type 1 diabetes often feel hesitant toward engaging in physical activity (PA) due to the risk of postexercise hypoglycemia. A blunted epinephrine response makes nocturnal hypoglycemia $(\mathrm{NH})(7-11 \mathrm{~h}$ postexercise) particularly hazardous and common in youth who engage in afternoon exercise (4). The aim of this study was to identify the frequency of $\mathrm{NH}$ in a young population of individuals with well-managed type 1 diabetes (T1D), who were using either standard openloop control (OL) or a closed-loop (CL) insulin delivery system, with or without exercise in the previous afternoon.

\section{Methods}

This randomized, crossover study design involved 12 male and female subjects, 12-26 years of age, from the Yale T1D
Program. Subjects were required to have a minimum of 1-year clinical diagnosis of T1D and to be utilizing insulin pump therapy. On two separate occasions, subjects were admitted for $48 \mathrm{~h}$. One of the days was an exercise day in which treadmill running was completed in the afternoon $(3 \mathrm{pm})$ at $50-55 \%$ of maximal effort. These exercise sessions involved four $15 \mathrm{~min}$ bouts of physical activity with interspersed $5 \mathrm{~min}$ rest sessions. Intense physical activity was only permitted during these times. The second admission day was a sedentary resting day. The order of the days was randomized for each participant. On each of these 2-day admissions, meal content and times were kept constant and snacks were only allowed for the treatment of hypoglycemia. The OL control involved patients adhering to their "usual care" routine, using their preferred glucose meter and insulin pump from home with a new infusion set. Subjects determined their own insulin boluses for meals and also made appropriate modifications for exercise. The CL system involved two continuous glucose sensors (Medtronic Sof-Sensor; one of which served as a backup), a new insulin infusion set, a new study pump (Medtronic Paradigm 715), and a "Medtronic MiniLink REAL-Time transmitter adapted for 1 min transmission." An algorithm was set up on the study computer using 3-7 days of insulin usage data (Medtronic external PID algorithm). Radiofrequency signals allowed communication between the sensor and the pump. The algorithm's target glucose level was $120 \mathrm{mg} /$ day during the day and at night.

\section{Results}

Both sedentary (OL $135 \pm 46 \mathrm{mg} / \mathrm{dL}$ vs. CL $140 \pm 47 \mathrm{mg} / \mathrm{dL}$, $p=0.02)$ and exercise (OL $141 \pm 63$ vs. CL $147 \pm 56 \mathrm{mg} / \mathrm{dL}$, $p=0.04)$ conditions had lower $24 \mathrm{~h}$ mean blood glucose levels during OL versus CL control, a difference that was eliminated once readings $<70 \mathrm{mg} / \mathrm{dL}$ were disregarded. During exercise, there were no differences in the number of hypoglycemic episodes that required treatment between OL and CL therapy. On the nights following sedentary days, there was a greater percentage of $\mathrm{BG}$ values within the optimum $70-180 \mathrm{mg} / \mathrm{dL}$ range with CL control (98\% CL vs. $87 \% \mathrm{OL}$ ), and fewer incidences of $<70 \mathrm{mg} / \mathrm{dL}$ BG readings ( $4 \%$ OL vs. $1.5 \% \mathrm{CL}, p<0.0001$ ), which correspond to 8 (OL) versus 3 (CL) cases. On nights following afternoon exercise, OL treatment resulted in more hyperglycemic episodes and nocturnal low BG incidences increased by $11 \%$ when compared with CL control. In absolute terms, postexercise nights had 23 versus 11 cases of $<70 \mathrm{mg} / \mathrm{dL}$ BG for OL and CL, respectively $(p=0.03)$. Across the entire experiment, OL control had 14 episodes of treatable $\mathrm{NH}$ $(<60 \mathrm{mg} / \mathrm{dL})$, whereas 2 episodes were seen on nights with CL control $(p=0.04)$. On nights after antecedent afternoon exercise, there were 8 (in 4 subjects) versus 1 (in 1 subject) cases of treatable $\mathrm{NH}$ episodes in OL versus CL control, respectively. Regardless of the method of glycemic control on exercise days, the majority of all $\mathrm{NH}$ cases took place between $10 \mathrm{pm}$ and $2 \mathrm{am}$. This corresponds to the $7-11 \mathrm{~h}$ window predicted for postexercise hypoglycemia. Overall, there was less insulin delivery and lower plasma insulin levels on these nights with CL than with OL control (insulin delivery: $0.8 \pm 1.09$ units/h vs. $0.98 \pm 0.33$ units $/ \mathrm{h}, p=0.008$ and plasma insulin: $15.57 \pm 7.3 \mu \mathrm{U} / \mathrm{mL}$ vs. $17.06 \pm 6.76 \mu \mathrm{U} / \mathrm{mL}$; $p=0.06$ ). 


\section{Conclusion}

CL control allowed for significantly lower incidences of hypo-, treatable hypo-, and hyperglycemia during this experiment. Overnight glucose control during CL regulation on sedentary days was well maintained, with more people in an optimum range than in OL therapy. Moreover, the CL system achieved better BG recordings, irrespective of antecedent sedentary or exercise behavior. Facilitated by a feedback control system, the number of cases of $\mathrm{NH}$ decreased with lower insulin delivery between $10 \mathrm{pm}$ and $2 \mathrm{am}$.

\section{Comment}

The results of this study showing better glycemic management with CL control, regardless of that day's physical activity status (sedentary/exercise), are promising. CL control will be particularly beneficial for youth who do not necessarily adhere to a strict exercise regimen from day to day and who cannot forecast their exercise patterns. Rather than continuing with insulin basal rates designed for sedentary times, regular feedback control during exercise allows for an automated reduction in insulin delivery at hypoglycemia-vulnerable time ranges. Reducing the risk of hypoglycemia can help lessen the barrier to physical activity for youth with T1D, making further optimization of this technology extremely liberating and beneficial. Of course, these promising CL results depend upon the entire setup functioning correctly at all times-which the authors acknowledge in their discussion. Moreover, a number of safeguards (orthogonal sensors, implementation of rescue-glucagon doses) and improvements to existing technology (algorithms for different types of exercise, sensors, transmission systems, ease of patient use) are likely needed to better prepare this system for home use.

\section{Adding heart rate signal to a control-to-range artificial pancreas system improves the protection against hypoglycemia during exercise in type 1 diabetes}

\author{
Breton MD, Brown SA, Karvetski CH, Kollar L, \\ Topchyan KA, Anderson SM, Kovatchev BP
}

Center for Diabetes Technology, University

of Virginia, Charlottesville, VA

Diabetes Technol Ther 2014; 16: 506-11

\section{Background}

Hypoglycemia risk is a major challenge for good glycemic control in T1DM. Engaging in physical activity increases the risk for repeated exposures to low BG levels, an impaired counterregulatory response and ultimately hypoglycemiaassociated autonomic failure (HAAF). Exercise-induced hypoglycemia can occur during exercise, or in early and late recovery periods. Despite advancements in glycemic control brought forth by the artificial pancreas (AP), the risk of hypoglycemia during and right after exercise persists because of several physiological and technical challenges (5). A heart rate (HR) monitor can relay data to a closed-loop system regarding physical activity status. This study assessed whether a HR monitor can deliver the necessary information regarding physical activity to a control-to-range (CTR) system to prevent hypoglycemia during exercise.

\section{Methods}

Twelve of 13 recruited adult subjects (aged $38 \pm 3.3$ years with a history of T1DM for $23.6 \pm 4.4$ years) completed the study. These individuals had been using insulin pumps for $>6$ months and had glycated hemoglobin levels of $6.9 \% \pm 0.2 \%$. The experiment was composed of two back-to-back clinical study stays, each being 24-26 h in length. Each afternoon of admission involved a 30 min cycle ergometer exercise session at an intensity of 9-10 on the Borg 20 point rating of perceived exertion scale (i.e., moderate intensity aerobic exercise). The same three meals were offered at every admission, with lunch being served early to heighten the risk for hypoglycemia during afternoon exercise. Prior to admission, subjects received two continuous glucose monitoring devices (Dexcom SEVEN PLUS CGM) and a new insulin pump (Omnipod Insulin Management System, filled with Humalog insulin), and were trained to use the Android-running DiAs portable AP platform equipped with the appropriate algorithm. Subjects' smartphones linked the actions of the CGM and insulin pump. The control condition used only the CTR system, without inputting any measured HR data. In the experimental condition, data from a HR monitor informed subjects to manually press an "exercise button" whenever HR reached $>125 \%$ of resting HR (RHR). This activated the CTR + HR condition on the AP system. Alternatively, when the HR was reduced to $<125 \%$ RHR, then the button was pressed again to return to sole CTR control. The CTR algorithm is designed to predict alterations in metabolic control and adjust insulin delivery accordingly. The additional information provided by HR monitors allowed the system to lessen insulin delivery, and do so at an earlier time. Plasma glucose level was measured regularly to assess a low blood glucose index (a numerical description of the risk for hypoglycemia), the frequency of hypoglycemia (glucose $<70 \mathrm{mg} / \mathrm{dL}$, or sensation of hypoglycemia symptoms), the percentage of time in euglycemia $(70-180 \mathrm{mg} / \mathrm{dL})$, and the mean glucose level.

\section{Results}

The presence of HR monitor data allowed for smaller deviations from pre-exercise $\mathrm{BG}$ during exercise; the average maximum BG change of $-29 \mathrm{mg} / \mathrm{dL}$ was seen with CTR only and $-5 \mathrm{mg} / \mathrm{dL}$ with CTR $+\mathrm{HR}$. There were, however, no significant differences in average risk for hypoglycemia during exercise and in the recovery period. The CTR $+\mathrm{HR}$ group had an insignificant reduction in the frequency of hypoglycemia during exercise (none vs. 2; $p=0.16$; effect size $=0.45)$, during recovery $(1$ vs. $2 ; p=0.56$; effect size $=$ 0.18 ), and overnight (none vs. one; $p=0.32$; effect size 0.32 ), and also an insignificant increase in time in normoglycemia overall $(81 \% \pm 3.9 \%$ vs. $75 \% \pm 4.3 \%)$, during exercise $(91 \% \pm$ $7.3 \%$ vs. $85 \% \pm 10.3 \%)$, during recovery $(86 \% \pm 9.3 \%$ vs. $84 \% \pm 7.9 \%)$, and overnight $(89 \% \pm 7.1 \%$ vs. $84 \% \pm 9.2 \%)$ compared to the control group. The CTR + HR group had lower BG levels overall and during the overnight period, whereas mean BG was unchanged during exercise and recovery periods. 


\section{Discussion}

Using HR data, this portable AP system decreases the risk for hypoglycemia during and after exercise by lowering insulin delivery once a predefined HR threshold is achieved. Importantly, the protection against hypoglycemia was not at the expense of hyperglycemia; average glucose was actually lower, and still in the normoglycemic range.

\section{Comment}

As mentioned by the authors, the low power in this pilot study (12 subjects) resulted in few measurements reaching statistical significance. Nevertheless, it was found that adding an HR monitor to the AP system is a feasible implementation, and that further investigation of this combined system for the protection against hypoglycemia during and after exercise is well warranted. This is an important finding because HR monitors are common and easy to use. Since the initiation of CTR + HR control (i.e., when HR was $>125 \%$ of RHR) was manually inputted, it is difficult to account for potential false alarms in the event that the HR monitor was actually linked to the DiAs platform. Furthermore, it is difficult to extend the results of this study to all patients with T1D since the subjects were not at risk for cardiovascular events, nor were they on medications that lowered HR, making the sample unrepresentative of all T1DM patients. Nonetheless, incorporation of accelerometers into the setup as a measure of physical activity could be one way to address this problem (6). Finally, it is important to note that the results presented here are only for mild exercise, and these protective effects against hypoglycemia and hyperglycemia need to be tested for other exercise modalities. In the future, a fully automated system based on HR and perhaps other physiological measures (temperature, accelerometry, etc.) should make this technology a valuable way to manage glucose levels during exercise.

\section{Three 15-minute bouts of moderate postmeal walking significantly improve $24 \mathrm{~h}$ glycemic control in older people at risk for impaired glucose tolerance}

DiPietro $J^{l}$, Gribok $A^{2}$, Stevens $M S^{2}$, Hamm $L F^{l}$, Rumpler $W^{2}$

${ }^{1}$ Department of Exercise Science, George Washington University of Public Health and Health Services, Washington, DC; and ${ }^{2}$ Beltsville Human Nutrition Research Laboratory, U.S. Department of Agriculture, Beltsville, MD

Diabetes Care 2013; 36: 3262-68

\section{Background}

Among other health risks, transient postmeal hyperglycemia can ultimately progress to type 2 diabetes. This is a common occurrence in older adults, and the greatest extent of damage is often done in isolated postprandial bouts of high BG (7). Exercise can augment the effects of insulin on reducing BG levels, thereby providing an obvious effective therapy. The purpose of this study was to determine the dif- ference between several smaller bouts of postmeal exercise compared to one longer bout of exercise on postprandial and $24 \mathrm{~h}$ glucose control in older individuals.

\section{Methods}

Ten healthy male and female subjects at risk for impaired glucose tolerance (fasting glucose $105-125 \mathrm{mg} / \mathrm{dL}$ ), $\geq 60$ years of age, were involved in this study. All subjects participated in a total of three different randomly ordered 2-day protocols in a whole-room calorimeter, separated by 4 weeks. The first $24 \mathrm{~h}$ of the protocol served as the sedentary control day. The second day also involved inactivity, except for one of three treadmill walking regimens: $15 \mathrm{~min}$ duration, done 30 min after eating each meal; 45 min duration, done at 10:30 am; 45 min duration, done at 4:30 pm. All exercise was performed under supervision at 3 metabolic equivalent of task $\left(\right.$ METs $\left[\mathrm{MET}\right.$ value $\left.\left.=\mathrm{VO}_{2}\left(\mathrm{~mL}(\mathrm{~kg} \mathrm{~min})^{-1}\right) / 3.5\right]\right)$, as previously calculated by a treadmill test. CGM data allowed for analysis of $24 \mathrm{~h}$ average glucose concentrations, average $3 \mathrm{~h}$ postlunch glucose concentrations, and average $3 \mathrm{~h}$ postdinner glucose concentrations.

\section{Results}

There was a reduced insulin response in the middle of the day as levels of this hormone significantly decreased after each subsequent meal (postlunch: $37.9 \pm 33.4$ vs. postbreakfast: $59.4 \pm 53.5 \mu \mathrm{U} / \mathrm{mL} ; p<0.001 \&$ predinner: $7.4 \pm 3.4$ vs. prelunch: $14.2 \pm 13.4 \mu \mathrm{U} / \mathrm{mL} ; p<0.001)$. Changes in $0-30 \mathrm{~min}$ postprandial insulin levels declined as the day progressed; values were $60 \%$ after breakfast, $40 \%$ after lunch, and $54 \%$ after dinner $(p<0.01)$. Postmeal exercise prevented additional rises in postprandial glucose values and this was most prominent after $6 \mathrm{pm}$, and these postdinner exercise benefits lasted until 7 am the following morning. The favorable decrease in average $24 \mathrm{~h}$ glucose concentrations were comparable between three bouts of $15 \mathrm{~min}$ walking sessions $(-10 \%, p<0.03)$ and one morning 45 min walk $(-8 \%$, $p<0.05)$ compared to control conditions. $45 \mathrm{~min}$ afternoon exercise resulted in no significant difference in average $24 \mathrm{~h}$ glycemia. Postmeal exercise was the only protocol that lowered the $3 \mathrm{~h}$ glucose response to dinner compared to the control day. One $45 \mathrm{~min}$ bout of morning exercise had no effect on postdinner glucose, and sustained afternoon walking (predinner) increased the $3 \mathrm{~h}$ elevation in glucose associated with dinner when compared with the control day. A correlation was found between $24 \mathrm{~h}$ glycemia amelioration and better $3 \mathrm{~h}$ postdinner values $(r=0.88 ; p<0.001)$.

\section{Conclusion}

This study demonstrated that the timing and frequency of exercise can impact the glycemic response as the volume and intensity of exercise was kept constant under all protocols (45 min/day at 3.0 METS). There were a number of benefits associated with a 15 min walk done soon after a meal: it lowered $24 \mathrm{~h}$ glucose concentrations to a level that was comparable to one sustained $45 \mathrm{~min}$ bout of morning walking, and it was the only exercise protocol that improved $3 \mathrm{~h}$ postdinner glucose values. The correlation between improvements in both 24 and $3 \mathrm{~h}$ postdinner glucose values indicates that walking after dinner could be a beneficial 
activity in the betterment of overall daily glucose homeostasis. This is an important finding because such smaller doses of frequently performed low-moderate intensity exercise sessions can result in better adherence in older individuals.

\section{Comment}

This study showed that simply walking for 15 min soon after a meal results in marked improvements in glycemia in older persons at risk for glucose intolerance. Given the prevalence and high number of health complications associated with glycemic dysregulation and with aging, it is of extreme importance to find simple cost-effective solutions that are not considered too onerous for the older patient. As mentioned by the authors, an exercise intensity of 3.0 METS (a mild walk) aligns with what is recommended for older adults (8), and this study shows that even short walks, perhaps while completing daily chores, can improve glycemic control as good or better than prolonged activity. By providing exercise prescriptions that are cost-effective, accessible, and less intimidating to older individuals, healthcare providers facilitate the development of self-efficacy in their patients, thereby increasing the likelihood of long-term adherence and health benefits.

\section{Changes in physical performance in older women according to presence and treatment of diabetes mellitus}

Lee $C G^{1-3}$, Schwartz $A V^{4}$, Yaffe $K^{4}$, Hillier $T A^{3}$, LeBlanc $E S^{3}$, Cawthon $P M^{5}$, for the Study of Osteoporotic Fractures Research Group

${ }^{I}$ Research Service, Veterans Affairs Medical Center, Portland, OR; ${ }^{2}$ Department of Medicine, Oregon Health \& Science University, Portland, OR; ${ }^{3}$ Center for Health

Research, Kaiser Permanente Northwest, Portland, OR; ${ }^{4}$ Department of Epidemiology and Biostatistics, University of California at San Francisco, San Francisco, CA; and ${ }^{5}$ California Pacific Medical Center Research Institute, San Francisco, $C A$

J Am Geriatr Soc 2013; 61: 1872-78

\section{Background}

Older adults with diabetes mellitus (DM) are at risk of having falls while carrying out the activities of daily living (9). One way to prevent or treat the risk associated with functional decline is to assess measures of physical performance to find the reasons for physical impairment in individuals with DM. This longitudinal study analyzed grip strength and walking speed in a group of older women to assess whether DM and/or the use of the insulin sensitizers (IS) affect the changes in physical performance that accompany aging.

\section{Methods}

A cohort of 2,864 Caucasian and African American women $>65$ years of age who were initially involved in the prospective study of osteoporotic fractures were examined on two occasions, spaced 4-7 years apart. DM status or medication use was self-reported, and medications were brought to the research clinic for documentation. Metformin or thiazolidinediones were the only categorized IS. Each visit involved the averaging of two trials in which subjects were timed as they walked $6 \mathrm{~m}$ at their usual gait speed (usual gait), and rapid gait speed (rapid gait), and also completed two trials on a dynamometer in each hand (grip strength). Questionnaires were also used to account for other drugs, socioeconomic status, and additional health markers and behaviors.

\section{Results}

Women with DM had insignificantly higher grip strength (DM: $18.5 \pm 4.8$; no DM: $17.9 \pm 4.2 \mathrm{~kg} ; p=0.08$ ), but slower usual (DM: 0.90 \pm 0.18 ; no DM: 0.96 $\pm 0.18 ; p<0.001)$ and rapid gait (DM: $1.15 \pm 0.25$; no DM: $1.24 \pm 0.25 ; p<0.001$ ) speed when compared to nondiabetic women. Throughout the study period ( $~ 5$ years), a greater decline in usual and rapid walking speed was seen in women with DM compared to women who did not have DM. There was no difference in the loss of grip strength with time between these two groups. Women with DM taking IS (particularly those taking metformin) had less decline in usual walk speed than those not taking IS. Walking regularly did not significantly alter physical performance in the women in the study.

\section{Discussion}

While DM status did not result in a difference in grip strength losses, women with DM had more of a decline in walk speed compared to women without DM. The use of IS in women with DM led to a smaller drop in usual walk speed than women with DM who did not take IS. The greater functional losses in the lower versus upper extremities are important to note because maintenance of lower body strength is of greater importance when aiming to prevent falls and mobility problems.

\section{Comment}

This study is important because it emphasizes the need to measure functional outcomes and use such measurements to establish ways in which potential injuries for individuals with DM can be recognized and prevented. The use of IS, and in particular metformin, appeared to help attenuate the reduction in walking speed, perhaps by improving muscle function and/or by improving cognitive function and micro- and macrovascular health. As noted by the authors, the generalization and reliability of some results may be difficult to predict because this was a cohort of relatively healthy individuals involved in a longitudinal study with much of the data collected via questionnaires. Nevertheless, the use of IS, particularly metformin, to combat the age-associated decline in physical performance measures can be one way older individuals can combat the risk of mobility problems. An attenuated decline in gait speed may allow the elderly to remain confident in their functional capabilities, thereby promoting continual engagement in healthy living. 


\section{Feasibility of a portable bihormonal closed-loop system to control glucose excursions at home under free-living conditions for $\mathbf{4 8}$ hours}

van Bon $A C^{l}$, Luijf $Y M^{1}$, Koebrugge $R^{2}$, Koops $R^{2}$, Hoekstra $\mathrm{JBL}^{1}$, DeVries $J H^{1}$

${ }^{I}$ Department of Internal Medicine, Academic Medical Centre, Amsterdam, the Netherlands; and ${ }^{2}$ Inreda Diabetic B.V., Goor, the Netherlands

Diabetes Technol Ther 2014; 16: 131-36

\section{Background}

This study aimed to remove a current closed-loop (CL) glucose control system from the clinical research setting and test it for $48 \mathrm{~h}$ while subjects led their typical lifestyles at home.

\section{Methods}

In this crossover study, 11 subjects with T1D using continuous subcutaneous insulin infusion for $>6$ months were analyzed. The experiment was designed to compare openloop (OL) and fully automated bihormonal (insulin and glucagon) CL glycemic control systems that received no manual exercise or mealtime notices. First, under OL control, the CGMS $^{\circledR}$ System Gold ${ }^{\mathrm{TM}}$ Medtronic MiniMed was inserted, and subjects administered insulin as per their usual care. After a minimum of 3 weeks, under CL control, and after a $12 \mathrm{~h}$ optimization period in the research center, subjects carried on with their desired routine for $48 \mathrm{~h}$, at or around their home. These subjects wore 2 D-Tron + pumps for automated subcutaneous insulin and glucagon administration and 2 Medtronic CGM sensors. These tools were linked to a personal computer running the algorithm software. The algorithm's insulin sensitivity parameter was dependent on the rise and fall of postprandial glucose levels. Preset boluses of insulin and glucagon were given if BG did not remain at optimal levels $(\sim 6.5 \mathrm{mmol} / \mathrm{L})$. Hypoglycemia was defined as a BG reading $<3.5 \mathrm{mmol} / \mathrm{L}$ at which point an alarm alerted the subject to eat supplemental carbohydrates (CHO). The system was also equipped with other warnings (high glucose, pump occlusion alert, etc.). The main measurement of the study was to compare OL to CL control with respect to the amount of time in euglycemia $(3.9-10 \mathrm{mmol} / \mathrm{L})$ on a given experimental day (day 1 or 2 ).

\section{Results}

The amount of supplemental $\mathrm{CHO}$ needed, total insulin infused, and time spent in euglycemia and in hyperglycemia were similar between OL and CL control; however, the median time spent in hypoglycemia was significantly higher on the second day with CL than OL $(2.8 \%$ vs. $0 \%)$. Similarly, median glucose level was also lower on day 2 and during the night of day 2 with CL compared to OL control. Among other minor technical problems (occlusion alerts, high glucose alert, loss of wireless connection, etc.), the backup sensor was needed 16 times in CL control. There was, however, a significantly greater amount of median time spent in eugylcemia on the first day of CL control (97.1\%) compared to OL control $(69 \%)$.

\section{Discussion}

This study showed that a bihormonal CL system is feasible at home with comparable time spent in euglycemia as OL control and a significantly lower median glucose levels by day 2 of use. The reduction in median glucose levels on day 2 of use does come at the expense of more time in hypoglycemia, however.

\section{Comment}

This study should be considered a major technological breakthrough since it brings the bihormonal AP home from the research setting. One of the challenges in this study was the elevated number of hypoglycemic episodes during the second day of CL control. The authors comment that this was possibly due to CGM problems. Similarly, technical issues regarding cable and wireless connections between the devices used in this study highlight the importance of optimizing the setup for faultless functioning, perhaps via incorporation of multiple system parts into one device. While there is work to be done on the technical, safety, and practical size of the device, CL control is beginning to demonstrate improvements in control over OL systems. Implementation of a bihormonal system, in tandem with removing the CL setup outside of the research lab and into the home setting, takes us all a step forward in advancing this technology for home use.

\section{Hypoglycemia begets hypoglycemia: the order effect in the ASPIRE in-clinic study}

Garg $S K^{1}$, Brazg $R L^{2}$, Bailey $T S^{3}$, Buckingham $B A^{4}$, Slover $R H^{1}$, Klonoff $D C^{5}$, Shin $J^{6}$, Welsh $\mathrm{JB}^{6}$, Kaufman $\mathrm{FR}^{6}$

${ }^{1}$ University of Colorado Denver, Aurora, CO; ${ }^{2}$ Rainier Clinical Research Center, Renton, WA; ${ }^{3} A M C R$ Institute, Escondido, CA; ${ }^{4}$ Stanford University, Stanford, CA; ${ }^{5}$ Mills-Peninsula Health Services, San Mateo, CA; and ${ }^{6}$ Medtronic, Inc., Northridge, $C A$

Diabetes Technol Ther 2014; 16: 125-30

\section{Background}

Repetitive cases of hypoglycemia can lead to hypoglycemia-associated autonomic failure (HAAF). As an alternative to continued basal insulin delivery, as demonstrated recently in the ASPIRE-in-clinic study (10), automatic pump suspension at a predefined glucose threshold reduces the risk of hypoglycemia and does not promote rebound hyperglycemia in patients with T1D. The purpose of this study was to assess how antecedent hypoglycemia affects CSII threshold suspension (TS) technology.

\section{Methods}

This study examined 50 T1D subjects who exercised until plasma glucose reached $\leq 85 \mathrm{mg} / \mathrm{dL}$ (YSI analyzer) and then rested, and hypoglycemia was evaluated after the plasma glucose was $<70 \mathrm{mg} / \mathrm{dL}$. In TS experiments, 
insulin was automatically suspended for $2 \mathrm{~h}$ once the sensor glucose (SG) reached a threshold of $\leq 70 \mathrm{mg} / \mathrm{dL}$; in control experiments, basal insulin delivery continued. Subjects were randomly assigned to Group A (TS in period 1 ; control in period 2) or Group B (control in period 1; TS in period 2). This experiment had a crossover design with a 3-10-day washout period between trials. The primary measurements of the study were hypoglycemia duration (time between $50-70 \mathrm{mg} / \mathrm{dL}$ ) and severity (lowest BG value observed). To ensure subjects' safety, the experiment was stopped if a YSI glucose value exceeded 300 or decreased below $50 \mathrm{mg} / \mathrm{dL}$.

\section{Results}

Hypoglycemia duration was shorter $(107.8 \pm 71.2 \mathrm{~min}$ in period 1 vs. $171.5 \pm 67.0 \mathrm{~min}$ in period $2 ; p<0.01)$, recovery from low BG was faster, and glucose values were higher when TS control took place in period 1 (P1TS) rather than in period 2 (P2TS). The total duration of hypoglycemia preceding P1TS was significantly lower than P2TS (16.6 min vs. $204.6 \mathrm{~min} ; p<0.001$ ). Period 1 did not follow a phase of continued insulin delivery, but could come after a previously terminated experiment (BG $<50 \mathrm{mg} / \mathrm{dL}$ ). On the other hand, period 2 followed at least 1 former control period in which basal insulin continued to be delivered despite low BG. The control condition had a $4 \mathrm{~h}$ observation period in which subjects were hypoglycemic for the whole time, and there was no difference whether the control time was in $\mathrm{P} 1$ or $\mathrm{P} 2$. The percentage of subjects who were hypoglycemic was less during P1TS compared to P2TS.

\section{Discussion}

This study showed that recent bouts of hypoglycemia make it more difficult to recover from future hypoglycemic episodes even when TS is used. In period 1, in which patients had not had antecedent hypoglycemia, TS resulted in rapid normalization of BG levels when hypoglycemia occurred. Although the technology was still effective when recent hypoglycemia was initiated using exercise, the time and depth of duration was higher. The authors conclude that the effect of antecedent hypoglycemia should be taken into consideration in the design of future experiments assessing strategies to limit hypoglycemia.

\section{Comment}

This study highlights the importance of avoiding severe, prolonged episodes of hypoglycemia and the self-propagating cycle of glycemic dysregulation (i.e., "hypoglycemia begets hypoglycemia"). One should consider that a previous bout of exercise that promotes mild hypoglycemia makes TS technology less able to mitigate recovery hypoglycemia, unless the predefined thresholds are elevated. As the authors point out in their discussion, the implementation of the TS system immediately following a bout of hypoglycemia may help individuals with T1D speed up recovery from low BG and thereby better protect themselves from developing HAAF.

\section{Exercise prevents fructose-induced hypertriglyceridemia in healthy young subjects}

Egli $L^{l}$, Lecoultre $V^{l}$, Theytaz $F^{l}$, Campos $V^{l}$, Hodson $L^{2}$, Schneiter $P^{1}$, Mittendorfer $B^{3}$, Patterson $B W^{3}$, Fielding $B A^{2,4}$, Gerber $P A^{5}$, Giusti $V^{6}$, Berneis $K^{5}$, Tappy $L^{1,6}$

${ }^{1}$ Department of Physiology, University of Lausanne, Lausanne, Switzerland; ${ }^{2}$ Oxford Centre for Diabetes, Endocrinology and Metabolism, University of Oxford, Oxford, UK, ${ }^{3}$ Center for Human Nutrition, Washington University School of Medicine, St. Louis, MO; ${ }^{4}$ Department of Nutrition and Metabolism, University of Surrey, Guildford, UK, ${ }^{5}$ Division of Endocrinology, Diabetes, and Clinical Nutrition, University Hospital Zurich, Zurich, Switzerland; and ${ }^{6}$ Service of Endocrinology, Diabetes and Metabolism, Lausanne University Hospital, Lausanne, Switzerland

Diabetes 2013; 62: 2259-65

\section{Background}

Both rodent and human studies suggest that excess fructose (sugar/high-fructose corn syrup) can lead to a myriad of metabolic conditions, including type 2 diabetes, in part due to their ability to impact liver function (11). Among other benefits, exercise is known to improve lipid profiles such as reducing triglyceride-rich lipoprotein (TRL) and triglycerides (TG) levels in both lean and obese individuals (12). This study sought to examine whether exercise could improve the negative metabolic effects of a high-fructose diet.

\section{Methods}

Eight sedentary, healthy, nonobese males, aged 21.5 \pm 2.7 years, were involved in this crossover study. Subjects were studied on 3 separate 4-day protocols: low-fructose weight-maintenance diet, and no exercise (control); weight maintenance diet with high-fructose $(30 \%)$ served as lemonflavored beverages, and no exercise [HFr]; and high-fructose (30\%) diet plus $30 \mathrm{~min}$ of cycling exercise at $125 \mathrm{~W}$ power output at 12 and 5 pm every day [HFrEx]. Oral fructose tests $\left[{ }^{13} \mathrm{C}\right]$ were conducted on the 5 th day of each protocol.

\section{Results}

The HFr sedentary protocol led to a significantly elevated plasma TG, TRL-TG, apolipoprotein B48 (apob48), and glucose concentrations compared to the control group $(p<0.05)$. Exercise significantly decreased all of these changes compared to the HFr group, except fasting plasma glucose concentrations. Furthermore, the HFrEx group had reduced small LDL particles compared to control, and increased LDL- 1 and $\beta$-hydroxybutyric acid (BHB) compared to HFr. During oral fructose loading, the HFr trial resulted in elevated postload total TG, TRL-TG, $\left[{ }^{13} \mathrm{C}\right] \mathrm{TRL}-$ palmitate, and glucose concentrations, but decreased NEFA concentrations and lowered clearance of TRL-TG. With HFrEx, postprandial levels of total TG, TRL-TG, and $\left[{ }^{13} \mathrm{C}\right]$ TRLpalmitate concentrations were all comparable to the control group and TRL-TG clearance increased by $66 \%$ in comparison to the HFr trial. HFrEx subjects also had higher nonesterified fatty acids (NEFA) and glycerol concentrations compared with both control and HFr, decreased insulin 
concentrations and $\mathrm{CHO}$ oxidation, and increased net lipid oxidation. Energy expenditure in the HFrEx group was only higher compared to the control group, not the HFr group.

\section{Discussion}

High levels of fructose, alongside a weight-maintenance diet, negatively affects blood lipids, and exercise can ameliorate these alterations. In the HFrEx trial, harmful lipid parameters induced in the HFr condition were normalized.

\section{Comment}

Given the high prevalence of fructose in the North American diet, it is important to understand the metabolic consequences of such consumption. As the authors mention, it should be noted that the average American has a fructose intake that is $\sim 10 \%$ of their diet (13), making it likely that the study's daily $30 \%$ load overexaggerated the effects of this sugar on the body. Since each individual trial was of shorter duration, future experiments analyzing the cumulative effects of long-term fructose intake on lipid metabolism, with or without regular exercise, would be beneficial. Of great relevance to individuals with impaired lipid profiles or those at risk for type 2 diabetes, exercise can serve as a viable therapeutic to lower the negative metabolic parameters induced by high-fructose feeding. Further research can provide insight into the optimum dose of exercise that ameliorates such effects.

\section{Author Disclosure Statement}

M.C.R. has been an industry sponsored speaker for Medtronic, Sanofi, and Eli Lilly.

\section{References}

1. Bussau VA, Ferreira LD, Jones TW, Fournier PA. The 10-s maximal sprint: a novel approach to counter an exercisemediated fall in glycemia in individuals with type 1 diabetes. Diabetes Care 2006; 29: 601-6.

2. Tansey MJ, Tsalikian E, Beck RW, Mauras N, Buckingham BA, Weinzimer SA, Janz KF, Kollman C, Xing D, Ruedy KJ, Steffes MW, Borland TM, Singh RJ, Tambor- lane WV; Diabetes Research in Children Network (DirecNet) Study Group. The effects of aerobic exercise on glucose and counterregulatory hormone concentrations in children with type 1 diabetes. Diabetes Care 2006; 29: 20-25.

3. Colberg SR, Hernandez MJ. The Big Blue Test: effects of 14 minutes of physical activity on blood glucose levels. Diabetes Care 2013; 36: e21.

4. Tsalikian E, Mauras N, Beck RW, Tamborlane WV, Janz KF, Chase HP, Wysocki T, Weinzimer SA, Buckingham BA, Kollman C, Xing D, Ruedy KJ; Diabetes Research in Children Network Direcnet Study Group. Impact of exercise on overnight glycemic control in children with type 1 diabetes mellitus. J Pediatr 2005; 147: 528-34.

5. van Bon AC, Verbitskiy E, von Basum G, Hoekstra JBL, DeVries JH. Exercise in closed-loop control: a major hurdle. J Diabetes Sci Technol 2011; 5: 1337-41.

6. Stenerson M, Cameron F, Wilson DM, Harris B, Payne $\mathrm{S}$, Bequette BW, Buckingham BA. The impact of accelerometer and heart rate data on hypoglycemia mitigation in type 1 diabetes. J Diabetes Sci Technol 2014; 8: 64-69.

7. Chang AM, Halter JB. Aging and insulin secretion. Am J Physiol Endocrinol Metab 2003; 284: E7-12.

8. United States Department of Health and Human Services. 2008 Physical Activity Guidelines for Americans. Washington, DC, 2008, pp. 29-34.

9. Schwartz AV, Hillier TA, Sellmeyer DE, et al. Older women with diabetes have a higher risk of falls: a prospective study. Diabetes Care 2002; 25: 1749-54.

10. Bergenstal RM, Klonoff DC, Garg SK, Bode BW, Meredith M, Slover RH, Ahmann AJ, Welsh JB, Lee SW, Kaufman FR; ASPIRE In-Home Study Group. Thresholdbased insulin-pump interruption for reduction of hypoglycemia. N Engl J Med 2013; 369: 224-32.

11. Bizeau ME, Pagliassotti MJ. Hepatic adaptations to sucrose and fructose. Metabolism 2005; 54: 1189-201.

12. Hudgins LC, Hellerstein MK, Seidman CE, Neese RA, Tremaroli JD, Hirsch J. Relationship between carbohydrate-induced hypertriglyceridemia and fatty acid synthesis in lean and obese subjects. J Lipid Res 2000; 41: 595-604.

13. Vos MB, Kimmons JE, Gillespie C, Welsh J, Blanck HM. Dietary fructose consumption among US children and adults: the Third National Health and Nutrition Examination Survey. Medscape J Med 2008; 10: 160. 